\title{
Un modelo hedónico para los alquileres turísticos en la ciudad de Sevilla
}

\author{
SOLANO SÁNCHEZ, Miguel ÁNGEL \\ Universidad de Córdoba \\ Correo electrónico: d42sosam@uco.es \\ NúÑEZ TABALES, JULIA M. \\ Universidad de Córdoba \\ Correo electrónico: es2nutaj@uco.es \\ CARIDAD Y OCERIN, JOSÉ M. \\ Universidad de Córdoba \\ Correo electrónico: ccjm@uco.es
}

\begin{abstract}
RESUMEN
El auge de los nuevos tipos de alojamiento denominados alquileres turísticos (apartamentos turísticos y viviendas con fines turísticos) experimentado en los últimos años a nivel mundial, gracias a la expansión de Internet y las redes P2P, despierta el interés por el análisis de estas nuevas fórmulas de estancia. El objetivo de este estudio es la elaboración de un modelo a través del método de precios hedónicos (MPH) que establezca cuáles son las variables fundamentales en la determinación del valor diario de la estancia en alquileres turísticos. Dicha metodología, ha sido ampliamente usada en valoración inmobiliaria y determinación del precio en alojamientos turísticos (mayoritariamente hoteles), aunque se encuentran pocos trabajos en lo que respecta a su uso para valoración de alquileres turísticos. A partir de los datos recogidos sobre Sevilla -la mayor ciudad del sur de España- se obtiene un modelo econométrico para valoración de alquileres turísticos en el que destacan como principales variables explicativas del mismo la ubicación del inmueble, el tamaño, el equipamiento, así como el momento del tiempo en que es tomado el precio. Los resultados del presente estudio pueden resultar de interés tanto a propietarios como a potenciales clientes de alquileres turísticos, para conocer si el precio de la estancia está en concordancia con lo que normalmente ofrecería el mercado en esas circunstancias.

Palabras clave: alquileres turísticos, apartamentos turísticos, viviendas con fines turísticos, método de precios hedónicos, Sevilla.
\end{abstract}

Clasificación JEL: C51; C52.

MSC2010: 62P20.

Artículo recibido el 29 de abril de 2019 y aceptado el 9 de diciembre de 2019. 


\title{
A hedonic model for the vacation rentals in the city of Seville
}

\begin{abstract}
The boost of the accommodation type called vacation rentals (tourist apartments and dwellings) experienced worldwide in recent years thanks to the rise of the Internet and P2P networks arouses interest in the analysis of these new stay formulas. The aim of this research is the elaboration of an econometric model through hedonic pricing method (HPM) which establishes the fundamental variables in the determination of the daily rate in vacation rentals. This methodology, has been largely used in real estate valuation and price determination in tourist accommodation (mostly hotels), although scarcely regarding vacation rentals valuation. Through the data collected from Seville the largest city in southern Spain- an econometric model for valuation of vacation rentals is obtained in which stand out as main explanatory variables the location of the property, size, amenities, as well as the time period which the price is taken. The results of the present study could interest both owners and potential clients of vacation rentals, in order to know if the daily rate is in accordance with what would normally offer the market under those circumstances.
\end{abstract}

Keywords: vacation rentals, touristic apartments, touristic dwellings, hedonic pricing method, Seville.

JEL classification: C51; C52.

MSC2010: 62P20.

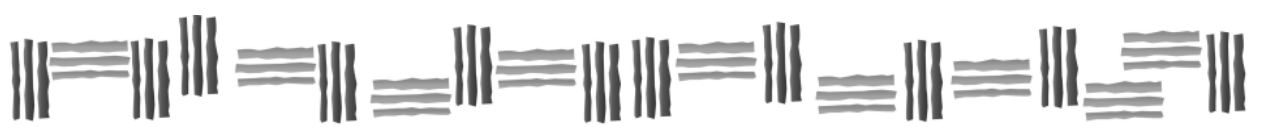




\section{Introducción.}

Los últimos diez años han supuesto un absoluto cambio de paradigma en el sector de alojamientos turísticos a nivel mundial. Frente a los convencionales alojamientos turísticos -tales como hoteles-, surge una nueva modalidad denominada alquileres turísticos. Este tipo de alojamiento puede definirse como la cesión temporal mediante precio de inmuebles privados que se ofrecen durante un tiempo determinado con un fin de explotación turística (De la Encarnación, 2016).

Factores como el auge de Internet han permitido a las plataformas peer-to-peer $(P 2 P)$ actuar como intermediarias y poner en común a millones de compradores y vendedores en el mundo (Gwin, 2004). En este caso particular, webs $P 2 P$ como Booking o Airbnb, han reunido a potenciales clientes y propietarios de alquileres turísticos de forma masiva. Este hecho, unido a ciertas ventajas que dicho alojamiento turístico presenta frente a los alojamientos convencionales, tales como mejor relación calidad/precio, disponibilidad de cocina y mayor privacidad (Statista, 2016), son las causas de su expansión como fenómeno global.

Por consiguiente, resulta de interés tratar de determinar los factores que permiten explicar el precio de estos nuevos tipos de alojamientos. Para ello, se seguirá el método de precios hedónicos (MPH), ya que se encuentra abundante literatura referida al uso de dicha metodología como sistema de valoración y predicción del precio de la estancia con respecto a alojamientos convencionales (establecimientos hoteleros, principalmente). El MPH considera el valorar un bien que está formado por distintos atributos o características que son inseparables; es decir, que deben valorarse en su conjunto (Rosen, 1974). Los modelos econométricos se han usado para este fin en diversos contextos, siendo la valoración de pisos y casas un ejemplo clásico. Sin embargo, la bibliografía existente en sistemas de valoración para alquileres turísticos mediante esta metodología resulta bastante escasa. Hasta la fecha, no se encuentran estudios referidos a sistemas de valoración en alquileres turísticos mediante MPH que utilicen a Booking como principal fuente de datos. La elección de Sevilla como ámbito de estudio es debido a que esta urbe es la mayor ciudad del sur de España y ha experimentado un notable aumento de este tipo de alojamientos en los últimos años (véase Apartado 3).

El objetivo de este trabajo es, por tanto, la elaboración de un modelo MPH relevante, que refleje de una manera lo más ajustada posible qué variables influyen en la composición del precio de la estancia por día en alquileres turísticos. Para su elaboración, se seleccionan datos de una ciudad de especial significancia turística como Sevilla, obtenidos a partir de diversas fuentes, tales como el Registro Andaluz de Turismo (RTA), Booking y Google Maps, entre otras. El tratamiento de la información se realiza a través de los programas informáticos IBM SPSS Statistics 25 y EViews 10.

Los alquileres turísticos objeto de estudio adoptan en Sevilla (mediante legislación autonómica andaluza, véase Apartado 3) la forma legal de apartamentos turísticos (AT) y viviendas con fines turísticos (VFT). Aunque ambos alojamientos consisten en inmuebles independientes, con las amenidades propias de una vivienda (como disponibilidad de cocina y cuarto baño propio), su diferencia principal reside en la modalidad de su registro: mientras que los AT son grupos de apartamentos (generalmente adosados unos a otros, aunque no necesariamente) que ocasionalmente disponen de zonas comunes (como recepción), las VFT se inscriben individualmente como unidades de alojamiento, pudiendo ofrecer la modalidad de estancia completa o por habitaciones. Si bien por la forma de explotación comercial (más empresarial) los AT guardan más similitudes con los alojamientos convencionales, habitualmente se engloban dentro de los alquileres turísticos (siendo la consideración efectuada a lo largo de este trabajo) por su gran parecido y su práctica equivalencia en comodidades ofrecidas al consumidor con las VFT (explotadas comercialmente a un nivel particular).

Con respecto a la estructura de este estudio, se presenta en primer lugar una revisión bibliográfica de investigaciones similares realizadas anteriormente (Apartado 2), para posteriormente dar paso a una concreción del ámbito de aplicación y objeto de estudio (Apartado 3), así como las variables inicialmente consideradas para la elaboración del modelo (Apartado 4). Finalmente, se muestran los 
resultados obtenidos (Apartado 5), valorando la utilidad de lo logrado y exponiendo las conclusiones alcanzadas (Apartado 6).

\section{Revisión bibliográfica.}

La idea subyacente en el MPH (método de precios hedónicos), según Parker y Zilberman (1993) es que el precio de un bien está formado por un agregado del valor de una serie de características que influyen en éste. Estas características no son divisibles, sino que se ofrecen como un todo, de forma que el demandante tiene que aceptarlas o rechazarlas en su conjunto. Desde el punto de vista econométrico, estos atributos no son, generalmente, independientes entre sí, lo que suele provocar problemas serios de multicolinealidad, que obligan, bien a utilizar técnicas no lineales, técnicas de reducción de dimensión como la de mínimos cuadrados parciales o de regresión en componentes, o bien generar índices que recojan grupos de características. De esta forma, conociendo individual y pormenorizadamente esa serie de atributos, así como su ponderación en la determinación final del bien analizado mediante el uso de técnicas estadísticas de regresión, se obtiene una estimación del precio del bien objeto de análisis asignando valores concretos a las variables que influyen en él.

La mayoría de autores establecen el origen del MPH a partir de los trabajos de Court (1939). Éste, fue el primero en utilizar el término "hedónico" en su estudio acerca de la determinación del aumento de precio de los automóviles. Posteriormente, autores como Griliches (1971) y especialmente Rosen (1974) desarrollaron un tratamiento unificado del MPH, planteando modelos de oferta y demanda de bienes heterogéneos, formados por diferentes atributos, y sentando las bases del enfoque más comúnmente aceptado, popularizándose su uso a partir de entonces en multitud de diferentes aplicaciones, entre las que destacan las del ámbito inmobiliario.

Dado que el objeto de este trabajo se basa en el análisis del precio de un tipo de inmueble vinculado al sector turístico, a continuación serán examinados los estudios previos que han aplicado el MPH tanto en la valoración de inmuebles como en el ámbito de alojamientos turísticos, especialmente aquellos trabajos dedicados a la estimación del precio de las estancias en hoteles, así como las variables que lo determinan.

Con respecto a los trabajos de valoración en vivienda a través del MPH a nivel internacional, son de especial relevancia los de Freeman (1979) -al utilizar la primera justificación teórica del uso del MPH al mercado inmobiliario-, Palmquist (1980) para el caso de King County (Washington, EE.UU.) y Kang y Reichert (1991) para Chicago (EE.UU.). A nivel nacional, son destacables los de Caridad y Brañas (1996), Caridad y Ceular (2001), García-Pozo (2005), Núñez (2007), Muñoz (2012) y Núñez et al. (2013) en el que se realiza una comparación con otra metodología como las redes neuronales artificiales. Pérez-Priego et al. (2019) utilizan técnicas de segmentación que son trasladables para categorizar las propiedades. Posteriormente, destacan los sistemas de valoración según MPH en locales comerciales y naves industriales de Rey (2014) y Casas et al. (2018), respectivamente. En trabajos referidos a la comunidad autónoma andaluza, resaltan los estudios dedicados al turismo rural. En éste área, es reseñable el sistema de indicadores que Blancas et al. (2011) proponen en su estudio para analizar la sostenibilidad del turismo rural en dicha región.

Sin embargo, dentro del terreno de la estimación de precio en alojamientos turísticos, es en la modalidad de hotel en donde se encuentran un mayor número de estudios realizados. A nivel internacional, destacan los trabajos de Chen y Rothschild (2010) para Taipéi (Taiwán), Andersson (2010) para Singapur, Zhang et al. (2011) para Nueva York (EE.UU.) y Kuminoff et al. (2010) para Virginia (EE.UU.) en donde se revelan las diferencias de precios entre hoteles convencionales y los "sostenibles" o "verdes".

A nivel nacional, caben mencionar los trabajos relacionados con el turismo de "sol y playa" como los de Coenders et al. (2003), Espinet et al. (2003) y, posteriormente, Rigall et al. (2011) centrados en 
la costa mediterránea en general y en Cataluña en especial. Merece ser destacado por seleccionar la misma ciudad que el presente estudio el análisis de Soler y Gémar (2017) acerca de la incidencia de la Feria de Abril en el precio de los hoteles de Sevilla. Recientemente, es reseñable también el estudio de estos mismos autores (entre otros) acerca de los condicionantes del precio de las estancias en los hoteles para la región portuguesa de Algarve (Soler et al., 2019).

En el campo de los alquileres turísticos, existen algunos estudios previos a nivel internacional. Mención especial merecen los trabajos de Dogru y Pekin (2017) acerca de los determinantes de valoración de las propiedades ofertadas en Airbnb para la ciudad de Boston (EE.UU.); así como el estudio hedónico de Gibbs et al. (2018) sobre alquileres turísticos en varias áreas metropolitanas de Canadá con datos obtenidos también a través de Airbnb. A nivel nacional, es pionero el estudio de Pérez y Marmolejo (2014) en el que se ofrece una comparativa de dos modelos hedónicos: uno para valoración de viviendas para residentes frente a otro de viviendas para turistas en la ciudad de Barcelona. Por lo tanto, puede afirmarse que la literatura referida a valoración de alquileres turísticos basada en MPH actualmente es aún muy reciente y exigua.

La Tabla 1 resume las aportaciones anteriormente mencionadas referentes a alojamientos turísticos ordenadas cronológicamente, indicando el año de la publicación, su objeto de estudio, las principales variables explicativas y la zona geográfica en la que se efectuó el análisis.

Tabla 1. Principales aportaciones al MPH en el ámbito de alojamientos turísticos.

\begin{tabular}{|c|c|c|c|c|}
\hline Autor/es & Año & Aplicación & $\begin{array}{l}\text { Variables utilizadas más } \\
\text { reseñables }\end{array}$ & $\begin{array}{l}\text { Localización del } \\
\text { análisis }\end{array}$ \\
\hline Coenders et al. & 2003 & $\begin{array}{l}\text { Precio de estancia en } \\
\text { hoteles }\end{array}$ & Mes del año, playa y parking & $\begin{array}{l}\text { Costa Mediterránea, } \\
\text { España }\end{array}$ \\
\hline Espinet et al. & 2003 & $\begin{array}{l}\text { Precio de estancia en } \\
\text { hoteles }\end{array}$ & $\begin{array}{l}\text { Piscina, playa, jardín y } \\
\text { localización céntrica }\end{array}$ & $\begin{array}{l}\text { Provincia de Gerona, } \\
\text { España }\end{array}$ \\
\hline Andersson & 2010 & $\begin{array}{l}\text { Precio de estancia en } \\
\text { hoteles }\end{array}$ & $\begin{array}{l}\text { Categoría del hotel }\left(\mathrm{n}^{\mathrm{o}} \text { de }\right. \\
\text { estrellas), } \\
\text { localización y piscina }\end{array}$ & Singapur \\
\hline $\begin{array}{l}\text { Chen y } \\
\text { Rothschild }\end{array}$ & 2010 & $\begin{array}{l}\text { Precio de estancia en } \\
\text { hoteles }\end{array}$ & $\begin{array}{l}\text { Localización céntrica, metros } \\
\text { cuadrados, piscina y televisión }\end{array}$ & Taipéi, Taiwán \\
\hline Kuminoff et al. & 2010 & $\begin{array}{lr}\begin{array}{l}\text { Diferencia de } \\
\text { entre }\end{array} & \begin{array}{r}\text { precios } \\
\text { hoteles }\end{array} \\
\text { convencionales } & \mathrm{y} \\
\text { "ecológicos" } & \end{array}$ & $\begin{array}{l}\text { Categoría del hotel }\left(\mathrm{n}^{\mathrm{o}} \text { de }\right. \\
\text { estrellas }) \text { cocina, Internet, } \\
\text { Jacuzzi, piscina y admisión de } \\
\text { mascotas }\end{array}$ & Virginia, EE. UU. \\
\hline Rigall et al. & 2011 & $\begin{array}{l}\text { Precio de estancia en } \\
\text { hoteles }\end{array}$ & $\begin{array}{l}\text { Distancia a la playa, tipo de } \\
\text { playa, Categoría del hotel ( }{ }^{\circ} \\
\text { de estrellas), parking, piscina } \\
\text { y mes del año }\end{array}$ & Costa Brava, España \\
\hline Zhang et al. & 2011 & $\begin{array}{l}\text { Precio de estancia en } \\
\text { hoteles }\end{array}$ & $\begin{array}{l}\text { Categoría del hotel, valoración } \\
\text { de usuarios, limpieza y } \\
\text { localización }\end{array}$ & $\begin{array}{l}\text { Nueva York, EE. } \\
\text { UU. }\end{array}$ \\
\hline $\begin{array}{l}\text { Pérez y } \\
\text { Marmolejo }\end{array}$ & 2014 & $\begin{array}{l}\text { Precio del } \mathrm{m}^{2} \text { por noche } \\
\text { en vivienda alquilada }\end{array}$ & $\begin{array}{l}\text { Barrio, Internet, } \\
\text { acondicionado, } \mathrm{n}^{\mathrm{o}} \\
\text { habitaciones }\end{array}$ & Barcelona, España \\
\hline Dogru y Pekin & 2017 & $\begin{array}{l}\text { Precio de estancia en } \\
\text { alquileres turísticos }\end{array}$ & $\begin{array}{l}\text { Valoración de usuarios, } \mathrm{n}^{\mathrm{o}} \text { de } \\
\text { fotos, admisión de mascotas, y } \\
\text { alojamiento completo/por } \\
\text { habitaciones }\end{array}$ & Boston, EE. UU. \\
\hline
\end{tabular}




\begin{tabular}{|c|c|c|c|c|}
\hline Soler y Gémar & 2017 & $\begin{array}{l}\text { Precio de estancia en } \\
\text { hoteles }\end{array}$ & $\begin{array}{l}\text { Categoría del hotel }\left(\mathrm{n}^{\mathrm{o}} \text { de }\right. \\
\text { estrellas), distancia al centro, } \\
\text { valoraciones de usuarios, día } \\
\text { de la semana durante la Feria } \\
\text { de Abril (Sevilla, España) }\end{array}$ & Sevilla, España \\
\hline Gibbs et al. & 2018 & $\begin{array}{l}\text { Precio de estancia en } \\
\text { alquileres turísticos }\end{array}$ & $\begin{array}{l}\mathrm{N}^{\mathrm{o}} \text { de plazas, distancia al } \\
\text { centro, parking, piscina } \mathrm{y}^{\circ} \text { de } \\
\text { fotos }\end{array}$ & Canadá \\
\hline Soler et al. & 2019 & $\begin{array}{l}\text { Precio de estancia en } \\
\text { hoteles }\end{array}$ & $\begin{array}{l}\text { Estrellas del hotel, } \mathrm{n}^{\mathrm{o}} \text { de } \\
\text { habitaciones, distancia al } \\
\text { centro, aire acondicionado, } \\
\text { etc. }\end{array}$ & Algarve, Portugal \\
\hline
\end{tabular}

Fuente: Elaboración propia.

\section{3. Ámbito de aplicación y objeto de estudio.}

El ámbito de aplicación del estudio es la ciudad de Sevilla, situada en el suroeste de España, en el centro de la provincia del mismo nombre. Con una superficie de $142,09 \mathrm{~km}^{2}$ (IDE Sevilla, 2018) y una población total de 689.434 habitantes (INE, 2017a) se erige como la mayor ciudad del sur de España.

Sevilla vive un momento turístico excepcional. El ayuntamiento estima que el sector supone un $17 \%$ del PIB de la ciudad (Barba, 2017), frente al 13\% a nivel andaluz (INE, 2017b) y el 11,7\% a nivel nacional (INE, 2017c). Es actualmente la tercera ciudad más visitada de España por turistas nacionales y la cuarta por turistas internacionales (INE, 2018). Además de ello, la prestigiosa guía Lonely Planet, eligió a esta ciudad como la número uno para visitar en 2018, a través de su publicación Best in Travel 2018 realizada en octubre de 2017 (Lonely Planet, 2017); asimismo, Sevilla fue la sede de la cumbre mundial celebrada por la organización internacional del sector turístico WTTC los días tres y cuatro de abril de 2019 (WTTC).

El objeto de estudio son los AT y VFT registrados legalmente en el municipio sevillano. La obligatoriedad de inscripción en el RTA (de acceso público y gratuito) que tienen los distintos tipos de alojamientos turísticos (AT y VFT incluidos), es establecida por la Ley 13/2011, de 23 de diciembre, del Turismo de Andalucía, en su artículo 37.

La citada ley define los AT (art. 44, ap. 1) como "aquellos tipos de alojamientos turísticos compuestos por un conjunto de unidades de alojamiento que cuentan con mobiliario e instalaciones adecuadas para la conservación, elaboración y consumo de alimentos y bebidas; que podrán ser según su tipología constructiva y configuración, apartamentos, villas, chalés, bungalós o inmuebles análogos".

Con referencia a las VFT, quedan reguladas a nivel autonómico andaluz a través del Decreto 28/2016, de 2 de febrero, de las viviendas con fines turísticos y de modificación del Decreto 194/2010, de 20 de abril, de establecimientos de apartamentos turísticos; que las define en su artículo tres como "aquellas ubicadas en inmuebles situados en suelo de uso residencial, donde se vaya a ofrecer mediante precio el servicio de alojamiento de forma habitual y con fines turísticos". Cabe destacar también los tipos de explotación establecidos (art. 5) para este tipo de alojamientos: bien en modo completo (limitándose a un máximo de quince plazas para este caso) o por habitaciones.

Por tanto, como punto de partida de la investigación, se toma como referencia la totalidad de registros de AT y VFT en el municipio sevillano aparecidos en el RTA obtenido en el momento de su solicitud (octubre de 2018). Esto será denominado a partir de ahora población. La muestra es considerada como aquella parte de la población de la que se han podido obtener datos completos relevantes para la elaboración del modelo pretendido. 
La Tabla 2 resume el número de registros totales (población) frente a los analizados (muestra). Con respecto al número de plazas, se procura una ponderación similar en muestra y población $(21,82 \%$ y $78,18 \%$ para AT y VFT respectivamente en población, frente a $26,90 \%$ y $73,10 \%$ de la muestra). En su conjunto, se ha obtenido una muestra que cubre más del $40 \%$ de la población.

Tabla 2. Población y muestra del estudio (octubre, 2018).

\begin{tabular}{lcccc}
\hline \multirow{3}{*}{ Población } & Tipo & $\mathbf{N}^{\mathbf{0}}$ de registros & Plazas & \% Plazas \\
& AT & 133 & 4.673 & $21,82 \%$ \\
& VFT & 3.465 & 16.739 & $78,18 \%$ \\
& Total & $\mathbf{3 . 5 9 8}$ & $\mathbf{2 1 . 4 1 2}$ & $\mathbf{1 0 0 , 0 0 \%}$ \\
\hline \multirow{3}{*}{ Muestra } & AT & 112 & 2.355 & $26,90 \%$ \\
& VFT & 665 & 6.401 & $73,10 \%$ \\
& Total & $\mathbf{7 7 7}$ & $\mathbf{8 . 7 5 6}$ & $\mathbf{1 0 0 , 0 0 \%}$ \\
\hline
\end{tabular}

Fuente: RTA (2018).

\section{Variables previamente consideradas.}

A partir de la revisión bibliográfica realizada en el apartado 2 se seleccionan una serie de variables que a priori se considera que pueden tener especial incidencia en el precio de la estancia en alquileres turísticos. Para la recogida de datos, se utiliza Booking como fuente principal, debido a que este portal refleja información pormenorizada de la vivienda, como los metros cuadrados de la propiedad o su ubicación exacta. Otras posibles fuentes -como Airbnb- no revelan las características del inmueble de forma tan detallada. A continuación, se procede a la descripción de los criterios de selección de las variables, así como del precio.

\subsection{Precio (PRE).}

La variable dependiente del modelo es tomada como precio por AT o VFT completas por día, para una estancia de dos noches, que es la estancia media para la ciudad de Sevilla según el Centro de Datos Turísticos del Ayuntamiento de Sevilla (2017). Se excluye del estudio la modalidad de VFT "por habitaciones", ya que distorsionaría el modelo conjunto al cuantificarse servicios diferentes a los ofrecidos por los AT. Además de ello, sólo supone un 7\% del registro total de VFT a nivel sevillano (RTA, 2018). La obtención de los precios ha sido posible a través de Booking (2018), siguiendo los criterios descritos a continuación:

- El precio siempre es tomado para una estancia de dos noches salvo cuando durante la obtención de datos la web fuerza a estancias mínimas superiores.

- El precio incluye impuestos y otros gastos añadidos. En las VFT se incluyen los suplementos de limpieza por estancia cuando procede.

- En el caso de aparecer varios precios por el mismo alojamiento, se escoge prioritariamente la opción de cancelación en plazo determinado y/o parcialmente reembolsable. La opción sin reembolso se selecciona únicamente cuando no existe otra opción posible.

- En caso de que un mismo complejo de AT oferte sus apartamentos al mismo precio, se escoge siempre aquel que por sus características ofrece un mayor valor añadido, tal y como se comporta cualquier consumidor racional. 


\subsection{Tipo (TIP).}

Variable binaria que es utilizada para distinguir los casos referidos a AT de las de VFT. La fuente de información usada para su obtención es el RTA (2018).

\subsection{Distrito (DIS).}

Registra el distrito de la ciudad de Sevilla (Figura 1) en donde se encuentra ubicado el alojamiento (Bellavista - La Palmera, Casco Antiguo, Cerro - Amate, Este - Alcosa - Torreblanca, Los Remedios, Macarena, Nervión, Norte, San Pablo - Santa Justa, Sur y Triana). Para su elaboración, se utiliza el programa informático Google Earth, mediante el archivo "SEVILLA_Distritos.xml" descargado a través de IDE Sevilla (2018).

Figura 1. Término municipal de Sevilla por distritos.

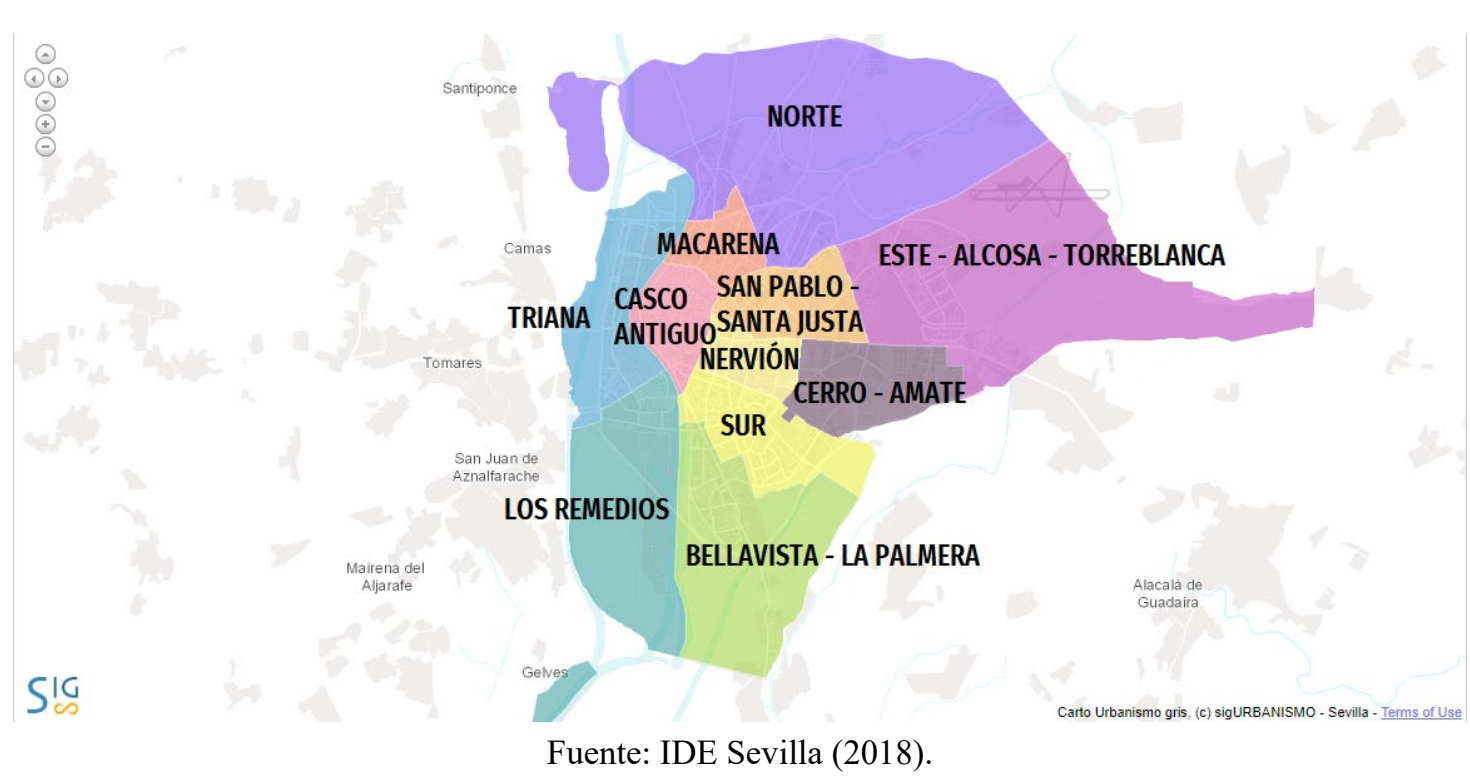

\subsection{Minutos caminando hasta la Plaza del Triunfo (MIN).}

Se consideran los minutos empleados caminando desde el alojamiento analizado hasta la Plaza del Triunfo de Sevilla. Este punto de referencia se encuentra entre la Catedral de Sevilla y el Real Alcázar, los dos monumentos más visitados en la ciudad en el año 2017 según el Centro de Datos Turísticos del Ayuntamiento de Sevilla (2017) y, por tanto, punto con mayor interés de acceso en el ámbito turístico.

Se plantea la hipótesis de que MIN actuará de manera inversamente proporcional: a más minutos empleados en llegar a la Plaza del Triunfo, el alojamiento tendrá un precio menor, es decir, cabría esperar que los alojamientos de mayor precio se encuentren más próximos a las zonas de mayor interés turístico. Para la obtención de la información, se usaron diferentes búsquedas a través de Google Maps (2018). 


\subsection{Número de plazas (PLZ).}

El número de plazas por el que se oferta el alojamiento. Conviene aclarar que un mismo alojamiento puede ofertarse con diferentes números de plazas, generando por tanto diferentes casos incluidos en el sistema -un total de 610 para AT y de 1623 para VFT-. Estos datos fueron obtenidos a través de Booking (2018).

\subsection{Metros cuadrados (M2).}

Los metros cuadrados construidos del AT o VFT se tomaron en su práctica totalidad a través de Booking (2018). Sin embargo, en la toma de tres casos, se acudió a la referencia catastral de la propiedad aparecida en la web Idealista (2018).

\subsection{Equipamiento (distintas variables).}

Se contabiliza por separado si el alojamiento dispone de televisión $(T V)$, lavadora $(L A V)$, balcón $(B A L)$, terraza $(T E R)$, patio $(P A T)$, vistas (VIS) (consideradas sólo las vistas a la ciudad y/o monumentos emblemáticos, nunca a patios y/o jardines interiores según lo observado en las fotografías disponibles en el perfil de la propiedad), insonorización (INS), parking (PAR) (considerando tanto del propio establecimiento como disponibilidad de parkings privados próximos), admisión de mascotas (MAS), piscina $(P I S)$ y bañera $(B A \tilde{N})$. Toda la información seleccionada en este apartado se obtuvo a través de Booking (2018).

\subsection{Valoraciones de anteriores usuarios (VAL).}

Booking ofrece la opción a las personas que hayan sido clientes de un alojamiento determinado de que valoren su experiencia, dando un resultado final numérico en una escala de cero a diez. Si bien es cierto que el cliente aquí está valorando más factores aparte del aspecto del alojamiento (tales como la ubicación, el personal, la limpieza o el equipamiento) priman las cuestiones relativas a la presencia y a la experiencia personal del cliente, por lo que se considera adecuado incluir esta variable dentro del modelo.

\subsection{Número de fotos publicadas (FOT).}

Booking permite a las propiedades subir fotos al perfil de las mismas. Se tiende a pensar que un mayor número de fotos implica un mayor deseo del propietario de hacer ver al potencial cliente el atractivo del alojamiento y viceversa: un número escaso de fotos podría ser debido a un intento del propietario de no proporcionar demasiadas muestras del insuficiente atractivo visual del alojamiento. Así, se cuantifica el número total de fotos publicadas en la propiedad como otra posible variable más en la composición de los modelos, que debería influir en su precio de una manera positiva.

\subsection{Atractivo Visual (AVI).}

Se incluye aquí una elaboración propia acerca del atractivo visual del alojamiento, tras el visionado de todas las fotos del perfil del alojamiento disponibles en Booking, cuantificándose como variable numérica de cero a diez. Se pretende ofrecer una valoración basada en criterios objetivos como el mobiliario, la limpieza, el orden y la calidad de las fotografías.

\subsection{Estacionalidad (AES, AFS, BES, BFS, SS y FE).}

Para la elaboración del modelo, el precio es tomado en distintos momentos del tiempo. Se han considerado seis intervalos de dos días (Tabla 3). Para la ponderación entre los distintos momentos del tiempo en los que el precio fue tomado, se considera una división aproximada en dos mitades iguales en cuanto a las temporadas alta (de abril a septiembre, seis meses) y baja (de octubre a marzo, seis 
meses) otorgando un peso mayor a la temporada alta, debido al consecuente aumento de las pernoctaciones en la misma $(47,74 \%$ frente a $39,77 \%$, respectivamente).

Con referencia a los fines de semana, suponen algo más de las dos séptimas partes de los casos si se comparan con los casos entre semana, debido al evidente aumento de las pernoctas en ese intervalo. Respecto a los periodos de Semana Santa $(S S)$ y Feria $(F E)$, de una semana de duración cada uno de ellos, se toma la referencia aproximada de cuadriplicar y doblar respectivamente el $2 \%$ que supone una semana sobre el total del año, debido al aumento de pernoctas que se realizan en estos intervalos concretos del año.

Tabla 3. Intervalos de tiempo considerados en la toma del precio y su ponderación.

\begin{tabular}{llcc}
\hline Fecha de la estancia & \multicolumn{1}{c}{ Descripción } & Casos & Porcentaje \\
\hline Del 27/05/19 al 29/05/19 & Temporada alta, entre semana $(A E S)$ & 758 & $33,95 \%$ \\
\hline Del 31/05/19 al 02/06/19 & Temporada alta, fin de semana $(A F S)$ & 308 & $13,79 \%$ \\
\hline Del 14/01/19 al 16/01/19 & Temporada baja, entre semana $(B E S)$ & 642 & $28,75 \%$ \\
\hline Del 18/01/19 al 20/01/19 & Temporada baja, fin de semana $(B F S)$ & 246 & $11,02 \%$ \\
\hline Del 18/04/19 al 20/04/19 & Temporada de Semana Santa $(S S)$ & 169 & $7,57 \%$ \\
\hline Del 10/05/19 al 12/05/19 & Temporada de Feria $(F E)$ & 110 & $4,93 \%$ \\
\hline & Total & $\mathbf{2 . 2 3 3}$ & $\mathbf{1 0 0 , 0 0 \%}$ \\
\hline
\end{tabular}

Fuente: Elaboración propia.

\subsection{Resumen de las variables consideradas.}

La Tabla 4 resume todas las variables recogidas para la elaboración del modelo, así como las fuentes usadas para ello. Se añaden estadísticos descriptivos obtenidos a través de la muestra (Tabla 4) para facilitar el análisis de la dimensión de la misma.

Tabla 4. Variables consideradas para el modelo y sus estadísticos descriptivos.

\begin{tabular}{|c|c|c|c|c|}
\hline Código & Variable & Media o \% & $\begin{array}{l}\text { Desviación } \\
\text { Típica }\end{array}$ & Fuente \\
\hline$P R E$ & $\begin{array}{l}\text { Precio (por día, para dos } \\
\text { días de estancia) }\end{array}$ & 161,47 & 99,091 & Booking (2018) \\
\hline TIP & Tipo (AT o VFT) & AT $(27,32 \%)$, VFT $(72,68 \%)$ & -- & RTA (2018) \\
\hline$D I S$ & Distrito & $\begin{array}{c}\text { Casco Antiguo }(84,77 \%), \\
\text { Triana }(9,67 \%), \text { resto } \\
(5,56 \%)\end{array}$ & -- & IDE Sevilla (2018) \\
\hline$M I N$ & $\begin{array}{l}\text { Minutos caminando hasta la } \\
\text { Plaza del Triunfo }\end{array}$ & 13,82 & 8,97 & $\begin{array}{l}\text { Google Maps } \\
\text { (2018) }\end{array}$ \\
\hline$P L Z$ & $\mathrm{~N}^{o}$ de plazas & 3,92 & 1,878 & \multirow{6}{*}{$\begin{array}{l}\text { Booking }(2018) \text { e } \\
\text { Idealista }(2018)\end{array}$} \\
\hline$M 2$ & Metros cuadrados $\left(\mathrm{m}^{2}\right)$ & 72,32 & 41,057 & \\
\hline$T V$ & Televisión & $99,37 \%$ & -- & \\
\hline$L A V$ & Lavadora & $90,28 \%$ & -- & \\
\hline$B A L$ & Balcón & $41,42 \%$ & -- & \\
\hline TER & Terraza & $34,89 \%$ & -- & \\
\hline
\end{tabular}




\begin{tabular}{|c|c|c|c|c|}
\hline$P A T$ & Patio & $29,74 \%$ & -- & \\
\hline$V I S$ & Vistas & $51,55 \%$ & -- & \\
\hline$I N S$ & Insonorización & $23,42 \%$ & -- & \\
\hline$P A R$ & Parking & $46,48 \%$ & -- & \\
\hline$M A S$ & Admisión de mascotas & $11,82 \%$ & -- & \\
\hline$P I S$ & Piscina & $4,39 \%$ & -- & \\
\hline $\boldsymbol{B} \boldsymbol{A} \tilde{\boldsymbol{N}}$ & Bañera & $28,66 \%$ & -- & \\
\hline$V A L$ & $\begin{array}{l}\text { Valoraciones de anteriores } \\
\text { usuarios }\end{array}$ & 8,847 & 0,653 & \\
\hline$F O T$ & $\mathrm{~N}^{\circ}$ de fotos publicadas & 35,68 & 11,256 & \\
\hline$A V I$ & Atractivo visual & 8,487 & 0,834 & Elaboración propia \\
\hline$A E S$ & $\begin{array}{l}\text { Temporada alta, entre } \\
\text { semana }\end{array}$ & $33,95 \%$ & -- & \multirow{6}{*}{ Booking (2018) } \\
\hline$A F S$ & $\begin{array}{l}\text { Temporada alta, fin de } \\
\text { semana }\end{array}$ & $13,79 \%$ & -- & \\
\hline$B E S$ & $\begin{array}{l}\text { Temporada baja, entre } \\
\text { semana }\end{array}$ & $28,75 \%$ & -- & \\
\hline$B F S$ & $\begin{array}{l}\text { Temporada baja, fin de } \\
\text { semana }\end{array}$ & $11,02 \%$ & -- & \\
\hline$S S$ & $\begin{array}{l}\text { Temporada de Semana } \\
\text { Santa }\end{array}$ & $7,57 \%$ & -- & \\
\hline$F E$ & Temporada de Feria & $4,93 \%$ & -- & \\
\hline
\end{tabular}

Fuente: Elaboración propia.

\section{Modelo propuesto y resultados obtenidos.}

Los modelos hedónicos pueden adquirir diversas formas funcionales. En principio no siempre existe una especificación óptima; la elección entre modelos alternativos se aborda de forma empírica. Por tanto, tras realizar varias pruebas con distintas especificaciones y transformaciones de variables, se elige la forma lineal, con la cual se realizan los distintos contrastes diagnósticos de validación del modelo.

En cuanto a las variables explicativas a incluir en el modelo hedónico, se prueba con diversas combinaciones alternativas -incluyendo interacciones entre las variables y términos cuadráticos-, eliminando aquellas variables que se mostraron no relevantes en la determinación del precio. Las variables finalmente incluidas en el modelo inciden en factores como la superficie, el número de plazas, la distancia a la zona turística, el atractivo visual, algunas características internas como el índice de atractivo visual y algún equipamiento extra, como el disponer de piscina; además de las variables que representan la estacionalidad de eventos singulares y el comportamiento cíclico semanal.

Algunas variables que a priori se pueden considerar importantes se han excluido tras realizar los contrastes estadísticos correspondientes. Destacan dos que podrían tener influencia, aunque no la muestran: el tipo de alojamiento y la puntuación dada por clientes anteriores. No se han detectado diferencias de valor entre viviendas (VFT) y apartamentos (AT), como se referirá en las conclusiones. Respecto a la opinión de valoración otorgada por visitantes anteriores, esta variable está relacionada con el índice de atractivo visual, por lo que, al incluir éste en el modelo, la valoración de clientes anteriores no ha resultado significativa. Alguna variable referida a equipamiento disponible tampoco ha resultado relevante; en algún caso se trata de equipamiento generalizado en la mayoría de las propiedades, lo que impide valorar su importancia. 
Los resultados que se detallan a continuación son aquellos para los que se obtiene un mayor grado de ajuste (porcentaje total de la variable dependiente explicada por el modelo sobre la varianza total de dicha variable). Además de ello, fueron excluidos del sistema dos casos atípicos de VFT considerados como no representativos ya que su precio diario superaba los $1.000 €$ y no corresponden a otras características como el tamaño o la ubicación de las plazas, y cuya inclusión distorsionaba significativamente el modelo. Los contrastes $\mathrm{T}$ son utilizados para contrastar la significación de cada uno de los parámetros del modelo (Caridad \& Caridad, 2018). Se obtiene en todos ellos una probabilidad límite $(p)$ inferior a 0,001 , por lo que son altamente significativos para niveles usuales, aceptándose por tanto la inclusión de las distintas variables explicativas. La Tabla 5 recoge los resultados descritos tales como los coeficientes del modelo propuesto, los estadísticos $\mathrm{T}$ junto con sus respectivas probabilidades límite $(p)$ y los factores de inflación de la varianza $(F I V)$ para comprobar que no existen relaciones importantes de multicolinealidad entre las variables explicativas.

Tabla 5. Variables del modelo lineal mediante MPH.

\begin{tabular}{lccccc}
\hline \multicolumn{1}{c}{ Variable } & Coeficiente & Error estándar & Estadístico T & $\boldsymbol{p}$ & $\boldsymbol{F I V}$ \\
\hline Constante & $-76,73$ & 12,915 & $-5,941$ & 0,000 & -- \\
$\boldsymbol{M I N}$ & $-1,161$ & 0,129 & $-9,012$ & 0,000 & 1,126 \\
$\boldsymbol{P} \boldsymbol{L} \boldsymbol{Z}$ & 13,679 & 0,716 & 19,093 & 0,000 & 1,525 \\
$\boldsymbol{M} \boldsymbol{}$ & 0,792 & 0,033 & 23,702 & 0,000 & 1,586 \\
$\boldsymbol{I} \boldsymbol{N S}$ & 12,656 & 2,655 & 4,767 & 0,000 & 1,067 \\
$\boldsymbol{P I S}$ & 33,712 & 5,398 & 6,245 & 0,000 & 1,032 \\
$\boldsymbol{A} \boldsymbol{V I}$ & 15,129 & 1,464 & 10,336 & 0,000 & 1,256 \\
$\boldsymbol{A F S}$ & 20,025 & 3,493 & 5,732 & 0,000 & 1,214 \\
$\boldsymbol{B} \boldsymbol{E} \boldsymbol{S}$ & $-29,225$ & 2,784 & $-10,498$ & 0,000 & 1,339 \\
$\boldsymbol{B} \boldsymbol{F S}$ & $-23,607$ & 3,788 & $-6,232$ & 0,000 & 1,187 \\
$\boldsymbol{S S}$ & 154,762 & 4,395 & 35,212 & 0,000 & 1,141 \\
$\boldsymbol{F E}$ & 127,115 & 5,257 & 24,182 & 0,000 & 1,092 \\
\hline
\end{tabular}

Fuente: Elaboración propia.

La primera observación que ha de hacerse es que, en el caso de la estacionalidad, se toma como base a $A E S$ (precio en temporada alta, entre semana) excluyéndose, por tanto, del modelo (Tabla 5). Los coeficientes de la Tabla 5 informan acerca de las variaciones marginales que se producen en el precio por cada una de las variables exógenas. En otras palabras, cada minuto $(M I N)$ extra empleado en caminar desde el alojamiento a la Plaza del Triunfo rebaja en 1,16€ el precio medio del mismo, de la misma forma que el hecho de que el alojamiento disponga de insonorización (INS) encarece el precio diario de la estancia en 12,66€. Finalmente, el precio diario de un alquiler turístico se encarecerá en $154,76 €$ en temporada de Semana Santa $(S S)$ con respecto al precio que tiene durante $A E S$.

Con respecto al ajuste del modelo, se obtiene un coeficiente de determinación del 70,8\% (es decir, el porcentaje de la varianza del precio que están explicando las variables independientes) y un error relativo medio $\pm 22,51 \%$. El error relativo medio muestra las diferencias en forma de porcentaje obtenidas entre las predicciones del precio por parte del modelo y los valores reales del mismo. Para comprobar la estabilidad de modelo, el test de Chow muestra la inexistencia de cambios estructurales tanto entre AT y VFT, como en el resto de los parámetros del modelo en varios subconjuntos de datos de la muestra.

Por último, la Figura 2 muestra una comparativa entre el precio real recogido durante el proceso de toma de datos y el valor estimado que proporciona el modelo conseguido. De existir un ajuste perfecto, la nube de puntos se mostraría como una línea diagonal exacta (Figura 2). La forma de la nube de puntos obtenida en la Figura 2 sugiere esta forma, especialmente hasta el rango de los $500 €$, en donde 
aumenta la variabilidad lo que dificulta su capacidad predictiva. Es decir, para las propiedades de mayor lujo, sería preciso incorporar alguna de sus características específicas no disponibles para el resto de las plazas.

Figura 2. Nube de puntos comparativa precio real vs. precio estimado.

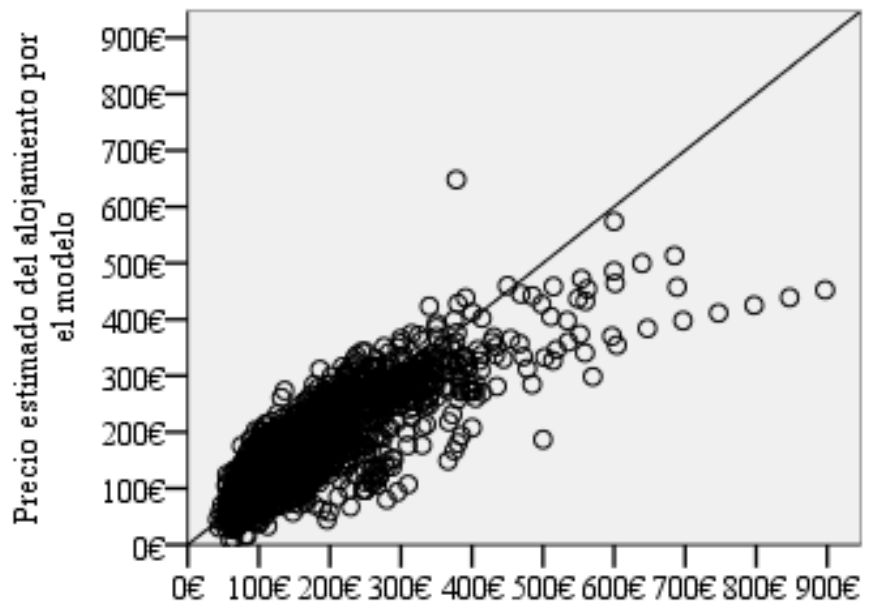

Precio real del alojamiento

Fuente: Elaboración propia.

\section{Conclusiones.}

Mediante la utilización del modelo obtenido pueden realizarse estimaciones ajustadas del precio de una estancia en alquiler turístico para la ciudad de Sevilla, para distintos tipos de propiedades y condiciones del entorno. Esto puede resultar interesante tanto a propietarios como a potenciales clientes de este tipo de alojamientos, para conocer si el precio de la estancia está en concordancia con lo que normalmente ofrecería el mercado para cada tipo de propiedad, con sus características específicas.

Con respecto a las variables exógenas calificadas como relevantes en la determinación de precios de alquiler, están en concordancia con las usadas en los modelos de estudios anteriormente mencionados, como la ubicación de la propiedad en relación a la zona turística o punto de interés, la superficie disponible, o algunas variables que definen propiedades de cada apartamento o vivienda, y que valoran los usuarios al tomar decisiones de reserva.

Estas variables, se incluyen en varias categorías: internas, externas y de temporalidad. Las internas de cada propiedad, como la superficie disponible para el huésped, algunas características específicas de la instalación, destacando la insonorización del piso y constatándose su influencia en el precio en el caso de disponer de piscina. Adicionalmente, la elaboración de un índice de atractivo visual en la presentación, ha resultado significativa en el modelo de valoración. Entre las externas destaca la ubicación medida no con criterios como la distancia calculada sobre coordenadas geográficas al núcleo de interés turístico sino propuesta como el tiempo de acceso a éste. Y finalmente, la estacionalidad es clara, tanto a nivel de grandes eventos turísticos, como intra-semanal.

Sin embargo, el tipo de alojamiento, es decir, la división entre AT y VFT no se muestra como determinante significativa del precio de alquiler. Si bien ambos registros se encuentran legalmente separados y desde el punto de vista del propietario se gestionan de manera diferente, desde la perspectiva del consumidor esto no se evidencia como aspecto relevante, ya que el servicio ofrecido en 
ambos (un alquiler de una unidad completa de alojamiento con cocina y cuarto de baño independiente) es prácticamente el mismo.

También es conveniente resaltar la multitud de variables potencialmente explicativas consideradas en el estudio no detectadas como relevantes, excluyéndose del modelo factores tales como el distrito (lo que es lógico, dado que se considera otra variable geográfica sobre la posición de la propiedad), las vistas -que son apreciadas una vez realizada la visita, pero que a priori son más difíciles de valorar en la plataforma donde el futuro cliente realiza su reserva-, la mayoría de las variables referidas al equipamiento, o la disponibilidad de aparcamiento, menos demandado por los visitantes que llegan mayoritariamente a la ciudad en transporte público.

Por último, como futuras líneas de trabajo se señalan la posibilidad de extrapolar los resultados a otras ciudades de especial relevancia turística, así como adaptar el modelo a otro tipo de alojamientos tales como los albergues. Y finalmente, la posibilidad de usar información parcial, como la referida del portal Airbnb y las opiniones de los clientes.

\section{Referencias}

Andersson, D.E. (2010). Hotel attributes and hedonic prices: an analysis of internet-based transactions in Singapore's market for hotel rooms. The Annals of Regional Science, 44(2), 229-240.

Barba, E. (2017, agosto 13). El turismo genera ya el 17\% de la riqueza de Sevilla, cinco puntos más que en España. Recuperado de https://sevilla.abc.es/sevilla/sevi-turismo-genera-17-por-cientoriqueza-sevilla-cinco-puntos-mas-espana-201708122121_noticia.html

Blancas, F.J., Lozano-Oyola, M., González, M., Guerrero, F.M., \& Caballero, R. (2011). How to use sustainability indicators for tourism planning: The case of rural tourism in Andalusia (Spain). Science of the Total Environment, 412, 28-45.

Booking (2018). Booking.com. Sevilla, Apartamentos + Casas. Recuperado de https://www.booking.com/searchresults.es.html?label=gen173nr1FCAEoggI46AdIM1gEaEaIAQGYAQq4ARjIAQzYAQHoAQH4AQuIAgGoAgQ\&sid=15def37 1e8a93ef38e3a7eada5b14ac4\&ac_click_type=b\&ac_position=0\&checkin_month $=5 \&$ checkin_mo nthday $=27 \&$ checkin_year $=2019 \&$ checkout_month $=$

Caridad, J.M., \& Brañas, P. (1996). Demanda de características de la vivienda en Córdoba: Un modelo de precios hedónico. Revista de Estudios Regionales(46), 139-153.

Caridad, J.M., Caridad, L. (2018). Estadística e Introducción al SPSS. Córdoba: Copisterías Don Folio.

Caridad, J.M., \& Ceular, N. (2001). Un análisis del mercado de la vivienda a través de redes neuronales artificiales. Estudios de Economía Aplicada, 18, 67-81.

Casas, J.C., Caridad, J.M., \& Núñez, J.M. (2018). La aglomeración urbana de la ciudad de Sevilla: análisis de la oferta de inmuebles destinados a la actividad comercial e industrial. Revista de métodos cuantitativos para la economía y la empresa, 25, 72-92.

Centro de Datos Turísticos del Ayuntamiento de Sevilla. (2017). Informe Anual 2017. Sevilla: Centro de Datos Turísticos del Ayuntamiento de Sevilla.

Chen, C-F., \& Rothschild, R. (2010). An application of hedonic pricing analysis to the case of hotel rooms in Taipei. Tourism Economics, 16(3), 685-694. 
Coenders, G., Espinet, J.M., \& Saez, M. (2003). Predicting random level and seasonality of hotel prices: a latent growth curve approach. Tourism Analysis, 8(1), 15-31.

Court, A.T. (1939). Hedonic price indexes with automotive examples. In General Motors Corporation, The Dynamics of Automobile Demand (pp. 99-117). Detroit: General Motors Corporation.

De la Encarnación, A.M. (2016). El alojamiento colaborativo: Viviendas de uso turístico y plataformas virtuales. Revista de Estudios de la Administración Local y Autonómica, 5, 30-55.

Decreto 194/2010, de 20 de abril, de establecimientos de apartamentos turísticos. Boletín Oficial de la Junta de Andalucía, no 90, de 11 de mayo de 2010, pp. 13 a 24.

Decreto 28/2016, de 2 de febrero, de las viviendas con fines turísticos y de modificación del Decreto 194/2010, de 20 de abril, de establecimientos de apartamentos turísticos. Boletín Oficial de la Junta de Andalucía, $\mathrm{n}^{\circ} 28$, de 11 de febrero de 2016, pp. 66 a 74.

Dogru, T., \& Pekin, O. (2017). What do guests value most in Airbnb accommodations? An application of the hedonic pricing approach. Boston Hospitality Review, 5(2), 1-13.

Espinet, J.M., Saez, M., Coenders, J.M., \& Fluvià, M. (2003). Effect on prices of the attributes of holiday hotels: a hedonic prices approach. Tourism Economics, 9(2), 165-177.

Freeman, A.M. (1979). The hedonic price approach to measuring demand for neighborhood characteristics. En S. D. Segal, The Economics of Neighborhood: Studies in Urban Economics (pp. 191-217). Nueva York: Academic Press.

García-Pozo, A. (2005). El mercado de la vivienda usada en Málaga. Una aplicación del modelo de precios hedónicos (Tesis Doctoral). Universidad de Málaga: España

Gibbs, C., Guttentag, D., Gretzel, U., Morton, J., \& Goodwill, A. (2018). Pricing in the sharing economy: a hedonic pricing model applied to Airbnb listings. Journal of Travel \& Tourism Marketing, 35(1), 46-56.

Google Maps (2018). Plaza del Triunfo. Sevilla. Recuperado de https://www.google.es/maps/place/Pl.+del+Triunfo,+41004+Sevilla/@37.3857238,$5.9944527,17 \mathrm{z} /$ data $=$ !3m1!4b1!4m5!3m4!1 s0xd126c199270f1 ff:0xaf2e9a0617c61dc9!8m2!3d37. $3856814 ! 4 \mathrm{~d}-5.9923465 ? \mathrm{hl}=\mathrm{es}$

Griliches, Z. (1971). Introduction: Hedonic Price Indexes Revisited en Price Indexes and Quality Changes: Studies in New Methods of Measurement. Cambridge: Harvard University Press.

Gwin, C.R. (2004). International Comparisons of Real Estate E-nformation on the Internet. Journal of Real Estate, 26(1), 1-24.

IDE Sevilla (2018). Distritos. Recuperado de http://sig.urbanismosevilla.org/visorgis/geosevilla.aspx?Layers=FOTOS\&Selected $=01 \&$ xtheme $=\mathrm{g}$ ray

Idealista (2018). Idealista.com, Calle Archeros 9 (y otras). Recuperado de https://www.idealista.com/archive/sevilla-sevilla/calle-archeros-9

INE (2017a). Cifras oficiales de población resultantes de la revisión del Padrón municipal a 1 de enero. Sevilla. Recuperado de http://www.ine.es/jaxiT3/Datos.htm?t=2911 
INE (2017b). Encuesta de Población Activa. Recuperado de Instuto de Estadística y Cartografía de Andalucía. Consejería de Economía y Conocimiento. Recuperado de http://www.juntadeandalucia.es/institutodeestadisticaycartografia/iea/resultadosConsulta.jsp?Cod Oper $=25 \& \operatorname{codConsulta}=110946$

INE (2017c). Aportación del turismo a la economía española. - Año 2017. Recuperado de https://www.ine.es/dyngs/INEbase/es/operacion.htm?c=Estadistica_C\&cid=1254736169169\&men $\mathrm{u}=\mathrm{ultiDatos} \& \mathrm{idp}=1254735576863$

INE (2018). Viajeros y pernoctaciones por puntos turísticos. Recuperado de http://www.ine.es/jaxiT3/Datos.htm?t=2078

Kang, H-B., \& Reichert, A.K. (1991). An Empirical Analysis of Hedonic Regression and GridAdjustment Techniques in Real Estate Appraisal. Real Estate Economics, 19(1), 70-91.

Kuminoff, N.V., Zhang, C., \& Rudi, J. (2010). Are travelers willing to pay a premium to stay at a "green" hotel? Evidence from an internal meta-analysis of hedonic price premia. Agricultural and Resource Economics Review, 39(3), 468-484.

Ley 13/2011, de 23 de diciembre, del Turismo de Andalucía. Boletín Oficial de la Junta de Andalucía, $\mathrm{n}^{\mathrm{o}} 255$, de 31 de diciembre de 2011, pp. 3 a 22.

Lonely Planet (2017). Lonely Planet's Best in Travel 2018. Melbourne: Lonely Planet.

Muñoz, G.A. (2012). Análisis de la vivienda en Córdoba.Estudio econométrico. (Tesis Doctoral). Universidad de Córdoba: España.

Núñez, J.M. (2007). Mercados inmobiliarios: modelización de los precios. (Tesis Doctoral). Universidad de Córdoba: España.

Núñez, J.M., Rey, F.J., \& Caridad, J.M. (2013). Precios implícitos en valoración inmobiliaria urbana. Revista de la construcción, 12(2), 116-126.

Palmquist, R.B. (1980). Alternative Techniques for Developing Real Estate Price Indexes. The Review of Economics and Statistics, 62(3), 442-448.

Parker, D.D., \& Zilberman, D. (1993). Hedonic estimation of quality factors affecting the farm-retail margin. American Journal of Agricultural Economics, 75(2), 458-466.

Pérez, V.B.. \& Marmolejo, C. (2014). El impacto de las externalidades producidas por el turismo sobre los valores inmobiliarios y la segmentación del mercado residencial en barcelona. $A C E$ : Architecture, City and Environment, 9(25), 159-188.

Pérez-Priego, M.A., García-Moreno, M.B., Gómez, G., \& Caridad, L. (2019). Segmentation based on the gastronomic motivations of tourists: the case of the Costa del Sol (Spain). Sustainability, 11(2), 409.

Rey, F.J. (2014). Alternativas y Determinantes en Valoración de Inmuebles Urbanos. (Tesis Doctoral). Universidad de Córdoba: España.

Rigall i Torrent, R., Fluvià, M., Ballester, R., Saló, A., Ariza, E., \& Espinet, J.M. (2011). The effects of beach characteristics and location with respect to hotel prices. Tourism Management, 32(5), 11501158. 
Rosen, S. (1974). Hedonic prices and implicit markets: product differentiation in pure competition. Journal of political economy, 82(1), 34-55.

RTA (2018). Registro de Turismo de Andalucía. AT y VFT. Sevilla: Consejería de Turismo y Deporte.

Soler, I.P., \& Gémar, G. (2017). Impact of the April Fair on Seville hotel room prices: measurement through a hedonic approach. Tourism \& Management Studies, 13(2), 7-12.

Soler, I.P., Gémar, G., Correia, M.B., \& Serra, F. (2019). Algarve hotel price determinants: A hedonic pricing model. Tourism Management, 70, 311-321.

Statista (2016). Leading reasons travelers stay in vacation rentals in the United States as of June 2016. Recuperado de https://www.statista.com/statistics/303324/most-common-reasons-for-travelers-tostay-in-vacation-rentals-us/

WTTC (2018, abril 19). 2019 WTTC Global Summit host announced: SEVILLE, SPAIN. Recuperado de https://www.wttc.org/about/media-centre/press-releases/press-releases/2018/2019-wttc-globalsummit-host-announced-seville-spain/

Zhang, Z., Ye, Q., \& Law, R. (2011). Determinants of hotel room price: An exploration of travelers' hierarchy of accommodation needs. International Journal of Contemporary Hospitality Management, 23(7), 972-981. 